\title{
¿Hay tiempo libre en la educación del tiempo libre? Etnografía a la Educación del Tiempo Libre
}

\author{
Gutiérrez Monclus, Pamela \\ Terapeuta Ocupacional. Licenciada en Ciencia de la Ocupación Humana. Diplomada en \\ Salud Mental. Doctorada (c) en Psicología Social en la Universidad Autónoma de \\ Barcelona. \\ Contacto > > Carrer Samanuego 41,2-2,CP:08035, Barcelona. email: \\ pamelagutierrezm@yahoo.es \\ Referencia > > Gutiérrez Monclus, Pamela. ¿Hay tiempo libre en la educación del \\ tiempo libre? Etnografía a la "educación del tiempo libre" en Barcelona.Revista Chilena \\ de Terapia Ocupacional. № 5, Noviembre 2005.
}

\section{- Abstract}

The present investigation puts attention to the normalization and discipline you present in "education in the free time" in Barcelona. This is an important area of the not formal education of a deep-rooted tradition.

The free time education, it has been a space defined from the promotion of the autonomy and whose automanagement take part in this, therefore, seems to be relevant to think to us brings over of the speeches and practices of control, which are given between members and especially, with the teenagers.

It is as well, as an ethnography has been realized in two centers of education of the free time ("Casal" and School Reinforcement), to think over respect of the forms ofgobernmentality in the education of the free time around four axes:

1. Actual practices

2. Categories that guide intervention

3. Periods of time and topics under consideration

4. Considerations by the researcher in the process of investigation

The conclusions wish to focus on the question, Is

\section{Resumen}

La presente investigación pone atención a la normalización y disciplina presentes en la "educación en el tiempo libre" ${ }^{\text {(i) }}$ en Barcelona, siendo esta un área de la educación no formal de una arraigada tradición.

La educación del tiempo libre, ha sido un espacio definido desde la promoción de la autonomía y la autogestión de quienes participan en ella, por lo tanto, nos parece relevante reflexionar acerca de los discursos y prácticas

de control, que se dan entre sus integrantes y en particular, con las ${ }^{\text {(ii) }}$ adolescentes.

Es así como se ha realizado una etnografía en dos centros de educación del tiempo libre (Casal y Refuerzo Escolar), para reflexionar respecto de las formas de gobernabilidad en la educación del tiempo libre en torno a cuatro ejes:

1.- las prácticas disciplinarias,

2.- categorías que guían la intervención,

3.- qué tipo de sujeto se busca construir,

4.- y los posicionamientos como investigadora en el proceso de investigación.

Las conclusiones buscan responder si ¿hay tiempo libre en la educación del tiempo libre? y 
there really free time during free time education?. And how is subject matter found and classified during the use of free time education with the teenagers? hacia que tipo de sujeto se busca "formar" en la educación del tiempo con las adolescentes.

(i) Trabajo de investigación que fue presentado como parte del Doctorado en Psicología Social de la Universidad Autónoma de Barcelona. Nombre de la investigación: " Discursos y Prácticas de Gobernabilidad hacia las Adolescentes. Aproximación Etnográfica a la Educación en el Tiempo Libre en Barcelona".

(ii) Para evitar el uso de masculino como genético (), sin complicar la lectura del texto añadiendo el femenino separado de una barra (), se ha optado por usar siempre el femenino, entendiendo que hacemos referencia al sustantivo elíptico (Las [personas] autoras]

\section{- Introducción "}

La investigación comenzó de la inquietud emanada de mi experiencia laboral durante cinco años de intervención comunitaria con niñas y adolescentes en la Municipalidad de La Cisterna, en la zona sur de Santiago de Chile. La experiencia de trabajo con adolescentes en la "Murga Aurora 2000"(iii), me llevó desde mi rol de Terapeuta Ocupacional y Jefa de la Unidad de Salud Mental a trabajar a la calle y así, participar de una experiencia de vida, de constantes encuentros cotidianos y de mucha sabiduría.

Hoy, ante el desafío del trabajo de investigación y recordando este cúmulo de experiencias, me interesa reflexionar, por una parte, sobre las formas en que la institucionalidad de la "educación en el tiempo libre" marca pautas, reglas, categorías de normalidad y disciplina respecto de las adolescentes. Y por otra, analizar cómo un ámbito caracterizado por la promoción de la autonomía y la autogestión, contiene formas de "subjetivación" y "gobernabilidad" hacia las adolescentes, al desarrollar sus propias intervenciones desde algunas nociones universales de "adolescencia".

Entenderemos por gobernabilidad ${ }^{(\text {iv })}$, al conjunto de prácticas por medio de las cuales, se pueden constituir, definir, organizar e instrumentalizar las estrategias que utilizan las personas libremente, para establecer relaciones unas con otras ${ }^{(18)}$. Por lo tanto, la gobernabilidad es un proceso que se basa en la libertad, expresada en la relación con el otro y consigo mismo. $Y$ por subjetividad ${ }^{(v)}$ entenderemos, a las distintas fuerzas y acciones que operan para que los seres humanos se constituyan en determinadas formas de sujeto, capaces a su vez, de tomarse como referentes de prácticas sobre sí mismos y los demás $\stackrel{(15)}{\text {. Por lo }}$ tanto, son los procesos que pautan el comportamiento de las personas de modo que se denominen a sí mismas y en relación con los demás como adolescentes.

Finalmente, cabe destacar que este trabajo de investigación ha sido construido colectivamente gracias a las diferentes reflexiones y aportaciones de valiosas personas ${ }^{(\mathrm{vi})}$. A su vez, explicitar mi rol en esta investigación como "interventora- intervenida", por estas experiencias, considerando que las reflexiones que realizo como investigadora respecto de la adolescencia, deben ser responsables y comprometidas con lo "situado y contingente" de éstas experiencias.

Por medio de esta investigación se busca conocer cómo se gestionan acciones hacia la adolescencia desde la "educación no formal"(vii) en el tiempo libre; cómo trabajan con las adolescentes, qué conceptos y qué categorías manejan estas instituciones y de qué modo éstas conceptualizaciones sobre la adolescencia, 
gobiernan la subjetividad de las adolescentes. Con el fin de ver en qué medida, "estas fantasías (...) son la claves de la regulación y subjetivación de los miembros" ${ }^{\prime 1}$

Objetivo General

- Conocer los discursos y prácticas de subjetivación y gobernabilidad hacia las adolescentes, mediante una aproximación etnográfica en dos centros de educación en el tiempo libre en Barcelona.

\section{Objetivos Específicos}

- Reflexionar acerca de las prácticas disciplinarias presentes en la educación del tiempo libre.

- Identificar desde qué categorías se realiza la intervención con las adolescentes en la educación no formal.

- Explorar qué tipo de sujeto (adolescente) desea configurar la educación en el tiempo libre.

Educación no formal y tiempo libre.

La educación no formal es un sector educativo disperso y heterogéneo, que como dice Trilla ${ }^{(2)}$ era considerado como un tipo de educación infravalorada académica, pedagógica y oficialmente; de modo que cualquier intervención educativa que no se realizara dentro de la escuela, quedaba marginada.

Desde 1970, en España, se reconoce la necesidad de desarrollar medios educativos diferentes a los convencionales, definiendo a éstos como "educación informal o no formal". Por ésta se entiende "un proceso que dura toda la vida y en el que las personas adquieren y acumulan conocimientos, habilidades, actitudes y modos de discernimiento mediante las experiencias diarias y su relación con el medio ambiente" ${ }^{\mathrm{(}}$ ) .

La tarea de la pedagogía del tiempo libre ${ }^{(\mathrm{viii})}$ según Trilla ${ }^{(2)}$, no es la de ocupar el tiempo libre mediante actividades formativas o instructivas; sino potenciar lo que de educativo tenga el ocio en sí mismo. Hace referencia al ocio como una actitud caracterizada por la libertad con el fin de conseguir el disfrute de la propia actividad y no en el producto de la misma.

El ideal de obtener "autonomía, participación y libertad" por parte de las adolescentes, está presente en los discursos institucionales que orientan las actividades de tiempo libre, pero dicho ideal está definido desde quienes gestionan estas actividades y con escasa participación de las adolescentes implicadas. También, en la pedagogía centrada en el niño, encontramos este ideal de potenciar un "niño libre, que aprenda por sí mismo".

La educación en el tiempo libre incluye diversas instituciones que cumplen una labor de "custodia", ofreciendo una alternativa a la "realidad problemática" del tiempo libre de las niñas y adolescentes. Es decir, la existencia de una parcela considerable de tiempo no ocupado por las instituciones educativas y de custodia tradicionales (familia y escuela), unida tanto a una constatación valorativa de que el uso que las niñas y adolescentes hacen de ese tiempo "no es el adecuado",y a la creencia pedagógica de que es posible intervenir institucionalmente en el uso del tiempo para optimizar formativamente su uso, se convierten en las justificaciones este ámbito educativo no formal.

Por tanto, nos encontramos ante la presencia, por un lado, del "ideal de libertad y autonomía" de las adolescentes en su tiempo libre; y por otro, de la comprensión de que el tiempo libre es un "área problemática", necesaria de intervención y guía. La profesionalización cada vez mayor de este espacio refleja como la "experticia" profesional se conecta con las metas del gobierno, en la formación de sujetos que se autodeterminen y autogestionen en las orientaciones que aparecen como deseables.

Inevitablemente nos surge la inquietud de si realmente ¿hay tiempo libre?, ¿cómo es posible la autogestión en un tiempo dirigido?, ¿cómo logran "hacer" las adolescentes en este entorno?, ¿qué categorías están 
presentes y qué sujeto se busca con la educación en el tiempo libre?. Preguntas que surgieron desde el trabajo de campo, guiando el presente trabajo y esperamos replantearlas en las conclusiones.

(iii) La Murga es un género popular carnavalesco que combina danza, música y canto, aunque este último puede estar o no presente (Punto Rock, 2005).

(iv) Foucault (19) se refiere a Gobernabilidad (Gobernmentality), como el "ensamblaje formado por instituciones, procedimientos, análisis y reflexiones, los cálculos y tácticas que permiten los ejercicios de esta muy específica aunque compleja forma de poder, que tiene como objetivo a la población, como su forma principal de conocimiento la economía política y como su medio técnico esencial a los aparatos de seguridad.La tendencia que, por un largo período y a través de occidente, ha sutilmente llevado a la preeminencia sobre toda otra forma (soberanía, disciplina, etc.) de este tipo de poder que puede ser denominado gobierno, resultando, por una parte, en la información de completas series de aparatos gubernamentales específicos, $y$, por otra parte, en el desarrollo de completos complejos de salvadores.Y al proceso, o más bien el resultado del proceso, a través del cual el estado de justicia de la edad media, transformado en un estado administrativo durante los siglos XV y XVI, gradualmente se vuelve gobernabilizado". (Foucault, 1991:102103).

(v) "Las tecnologías de la subjetivación, (...) son maquinaciones, además de instrumentos, componentes, entidades y aparatos prácticos e intelectuales, que producen ciertas formas de ser-humano, territorializaciones, estratificaciones, fijaciones, y vuelven perdurables las relaciones particulares que los humanos podrían realmente establecer consigo mismas" (15)

(vi) Se utilizará a partir de aquí -generalmente- la tercera persona plural, con el fin de reconocer las diferentes aportaciones a este trabajo.

(vii) Definición de educación no formal: "Conjunto de procesos medios e instituciones especificas y diferenciadamente diseñadas en función de objetivos explícitos de formación o de instrucción, no directamente dirigidos a la provisión de los grados propios del sistema educativo (Trilla, 1996 citado en Trilla y García, 2002).

(viii) Trilla (1993) cita indistintamente ocio y tiempo libre

\section{- Metodología: Etnografía Dialógica y Reflexividad"}

En la siguiente metodología nos hemos guiado por lo que dice Peter Spink ${ }^{(3)}$, ir "hacia un entendimiento de la etnografía que vincule la palabra acción". Y por medio de estas herramientas, generar un análisis que nos permita comprender los procesos de gobernabilidad y subjetivación en los entornos de la "educación en el tiempo libre" con las adolescentes.

En el proceso de definir la metodología de investigación, entendíamos que una aproximación etnográfica, contendría nuestra intención de poner atención tanto a los discursos cómo a las prácticas de las adolescentes. Junto a esto, buscábamos establecer una relación y no una separación entre investigadora y sujeto a quien se investiga, para así "trabajar sobre la base de la búsqueda de efectos de conexión, en relación con aquello que permita, desde nuestra posición de investigadoras, transformar nuestro punto de partida sobre el fenómeno a estudiar" (4). Esta investigación busca ser una construcción activa, y emergente desde las relaciones entre investigadora e investigada ${ }^{(5)}$.

Al comienzo la aproximación etnográfica tenía por objetivo colaborar en la definición del problema de investigación. Así, se comenzó la observación participante en el "Refuerzo Escolar" por al menos tres meses hasta definir la problemática. Continuando con la participación en el "refuerzo", se presentó la posibilidad de asistir a otro centro de educación en el tiempo libre, el "Casal". Desde este momento, desarrollé los diarios de campo, en ambas instituciones, hasta la finalización del período escolar.

La "llegada etnográfica" se realizó de diferentes maneras en los dos centros, en el "Refuerzo Escolar" ya existían relaciones previas y las entradas eran más confiadas. Sin embargo, fue necesario socializar la intención de la investigación, para poder ser monitora de grupos de adolescentes. En el "Casal" la sensación de estar abriendo múltiples puertas para integrarme, y poco a poco establecer alianzas, significó un largo 
periodo. Las relaciones con las adolescentes parecían frágiles y dependían de la proximidad y el trato, a su vez, debía respetar los tiempos -necesarios y diferentes- para lograr relaciones de mayor cercanía, lo que no dependía sólo, de quien investiga. Como citan Velasco y Díaz de la Rada, () "en esta tensión, y modo básico de aproximación al campo, se encuentra la observación participante". A su vez, fue fundamental alimentar la "capacidad de extrañamiento"(6), como una curiosidad hacia los distintos ámbitos, que podía llevarme la relación y que atraían mis intereses como investigadora.

Luego las entrevistas y conversaciones se mezclaron con los quehaceres diarios, realicé algunas entrevistas con algunas "informantes clave" con el fin de aclarar algunas dudas y profundizar en aspectos relevantes para la investigación. También fue posible realizar entrevistas colectivas de mucha utilidad. Como citan Velasco y Díaz de la Rada, ${ }^{(6)}$, "entrevistas" y "observación participante" son los dos tipos básicos de producción de información en el trabajo de campo. La observación participante, nos proporcionó el relato de los hechos como investigadora y las entrevistas, "tejidas sobre el diálogo" nos proporcionaron los relatos de con quienes nos relacionamos en esta investigación. Este proceso fue acompañado de las anotaciones en el diario de campo, tratando que entre la experiencia y la trascripción ocurriera el mínimo de tiempo posible ${ }^{(6)}$. El diario de campo se fue tiñendo de lecturas, conversaciones, tutorías y anotaciones al margen, permitiéndome recoger mis impresiones como investigadora y captar la investigación como situación ${ }^{(6)}$.

Un aspecto importante, fue la posibilidad de posicionarme desde distintos roles. En el "Refuerzo Escolar" pude participar como monitora, y encargada -junto a otras monitoras- de la última charla de padres. En el "Casal", realicé un rol de monitora según mi experiencia previa, luego me posicioné en un rol de colaboración con la monitora encargada del grupo, también colaboré como monitora de grupos de otras edades del Casal y finalmente (algunas veces) pude desempeñar algunos roles en los juegos de las chicas del Casal. Como citan Velasco y Díaz de Rada: "El etnógrafo se ha de esforzar porque su presencia sea de algún modo social, pero por otra parte, ha de saber admitir e interpretar, los roles que le son asignados por los agentes sociales, pues en esos roles se codifica parte de la información que el etnógrafo obtienen de la cultura" (6).

Finalmente, el trabajo de campo supuso la unificación de dos tareas, la recolección de la información y la elaboración teórica, por ello a nivel de análisis, el diario de campo se irá trabajando en torno a claves de la observación que emergieron en el transcurso de la investigación y tomaron cuerpo a través de la experiencia.

La intención del análisis es realizar un relato que dé cuenta, del espacio de relación, pudiendo relatar "mis experiencias" como investigadora miembro de un colectivo, y -en la medida de lo posible- las de las personas participantes, con el objeto de dar cuenta de la multiplicidad de voces. Aquí trabajaremos con el concepto de "etnografía dialógica" ( ${ }^{(7)}$ con el objeto de hacer presente lo "multivocal" y no sólo el mundo propio de quién investiga sino, la relación con las otras, buscando no hablar por las otras, ni hablar sólo por una misma, sino hablar acerca de la relación y las múltiples voces presentes en ella.

Las claves de observación tienen relación con los aspectos acerca de cómo se establecen formas de gobernabilidad hacia las adolescentes en torno a los siguientes ejes:

Cuales son las prácticas disciplinares hacia las adolescentes que participan en la educación en el tiempo libre.

Conocer desde qué conceptos y categorías, se trabaja con las adolescentes en la educación en el tiempo libre.

Perfilar a qué tipo de sujeto apunta la educación del tiempo libre y qué posibilidad de agencia y participación tienen las implicadas en estas actividades.

Localización

La selección de los lugares se dio en base a las posibilidades y conexiones que disponía al ser aún "recién llegada" a Barcelona, unido a mi experiencia previa en espacios de educación en el tiempo libre y al deseo de 
reflexionar acerca de las formas de gobernabilidad de estos espacios. Y también, sobre la valoración de las aperturas que pueden ofrecer estos centros, dirigidos a adolescentes con menores recursos económicos y promovidos desde el voluntariado y la solidaridad.

Los dos centros en que se realizó el trabajo de campo están vinculados a comunidades religiosas cristianas, basan sus acciones educativas más allá de la entrega de contenidos y buscan la entrega de valores y modelos de comportamiento. Ambas trabajan con el apoyo de voluntarias para llevar a cabo sus acciones. Estas organizaciones realizan coordinaciones territoriales con las demás organizaciones de su barrio.

El trabajo de campo comenzó en octubre del 2003, en el "Refuerzo Escolar" ubicado en un barrio de una cuidad colindante con Barcelona. Este barrio se caracteriza por un lugar de importante migración interna en los años 50 y actualmente recibe mucha migración magrebí y china, constituyendo un $20 \%$ de la población del sector. Participan un total de 45 niñas y adolescentes, de entre cinco a dieciocho años. Los grupos están divididos por edades, son mixtos y a cargo de monitoras. Las monitoras son voluntarias y la mayoría con formación profesional. Trabajé en el grupo de cinco chicas de 10 a 13 años, con dos monitoras más. Las actividades en este centro se realizan por las tardes, dos horas, una vez por semana.

Respecto del otro centro, la participación en el "Casal" comenzó en febrero del 2004. Éste se ubica en un barrio en el centro de Barcelona, que se caracteriza por ser un lugar antiguo y un centro importante de actividad comercial, fue sector de importante inmigración interna y actualmente recibe inmigración externa. También presenta una imagen desmejorada al ser casas antiguas y sin acceso a posibilidades económicas de mantención. En el Casal, participan un total de 110 niñas y adolescentes, de ellas 52 integran el Casal y el resto pertenece al área de fútbol sala. Trabajé con el grupo de 10 a 13 años en el que asistían entre 6 a 15 personas, las integrantes habituales son un grupo de 6 chicas con las cuales mantuve mayor relación, contaban con una monitora encargada del grupo. Las actividades se desarrollan de 17:00 a 20:00, de lunes a viernes, en las que participé como voluntaria una vez por semana.

\section{Resultados (x)}

\section{Dispositivo Modelo-Referente}

Al referirnos al "modelo-referente", buscamos mostrar cómo este dispositivo se instaura como un "modelo educativo" que encuadra tanto las relaciones de las monitoras y las alumnas, como las de los padres y sus hijos. El dispositivo "modelo-referente" en la relación monitora-alumna, estaba enmarcado en una relación personal, profesional y de entrega de valores morales. A su vez, al estar inscrita como una práctica de voluntariado, en el marco de la caridad cristiana, la relación monitora-alumna se establecía desde la lógica de ayudar y de dar al que no tiene.

Sumado a la inscripción de estas prácticas, en la lógica de la caridad, la relación monitora-alumna, se inscribe también en la profesionalización y la experticia, como dice Rose ${ }^{(8)}$ "una relación intrínseca con la autoridad de expertos" que permita gobernar a distancia por medio de "autoridades expertas autónomas". Es así cómo una parte importante de las voluntarias presentaba una maestría evidente, la mayoría contaba con formación universitaria o en proceso de su consecución. También ocurría que algunas monitoras, dada su formación profesional, contaban con un respaldo institucional, para desempeñar un rol de expertas, asesorando a las niñas con mayores dificultades y desempeñando un rol importante en la organizaciones de actividades dirigidas a los padres. La asesoría era de cara a las niñas o a los padres, no se ofrecía su "experticia" para un mejor desempeño de la labor de las monitoras o una revisión de los modelos educativos que ocupábamos. Cabe destacar, que aún cuando el equipo de monitoras recibió charlas de capacitación, no se nos entregó herramientas acerca de cómo nosotras -las monitoras-, podríamos resolver las dificultades en los procesos de enseñanza, lo que reforzaba la idea de que la incapacidad estaba puesta sólo desde las adolescentes. 
El dispositivo "modelo-referente"al basarseen la "díada, él que sabe y él que no sabe", permitía que se distribuyan como "verdad", los saberes expertos, que se han gestado en la matriz de la filantropía y la medicina ${ }^{(9)(10)}$. Esto posibilitaba una relación de superioridad de las monitoras respecto de las alumnas, volviendo a éstas sujetos de la relación de experticia. Lo que genera que unas sean "expertas" y a otras "necesitadas" y en donde la definición de roles de las personas, era fijada desde un lugar de poder ${ }^{(11)}$.

Sin embargo, en la práctica de esta "díada", había matices, en ocasiones, las monitoras no encontrábamos las respuestas adecuadas, por desconocimiento u olvido de las temáticas que abordaban las niñas. En esto, fue importante reconocer frente a las adolescentes, que no manejábamos esas temáticas y así mostrar que no sabíamos y pedir ayuda a una compañera o acudir a algún libro. Lo que también ocurría en ocasiones, era que aún existiendo el desconocimiento por parte de la monitora, la dificultad o falta era puesta sobre la adolescente que solicitaba la orientación, de modo que no se salía de la "díada él que sabe, él que no sabe", sino que se reforzaba, en la acentuación de la incapacidad del otro, sujeto de la relación de experticia.

A la relación de experticia, se sumaba que la coordinación contaba con un conocimiento acerca de las características de las adolescentes y de las monitoras que permitía por una parte, hacer una efectiva asignación entre monitoras-alumnas y por otra, prever que en los grupos de trabajo, se contara con monitoras con mayor experiencia, que guiaran a las monitoras nuevas en las prácticas institucionales. En ello, la regulación entre las monitoras, respecto de asistencia, puntualidad y desempeño, se volvían prácticas sutiles y efectivas de gobernabilidad de unas con otras.

Una de las características del dispositivo "modelo-referente" era que se establecía desde una relación individualizada, por ejemplo, de modo ideal se esperaba que por cada dos alumnas existiera una monitora, lo que permitía un control y regulación de las conductas y procesos de enseñanzas directamente. La individualidad del trato se reflejaba también, en la internalización de las prácticas disciplinarias por parte de las adolescentes, de hecho frente a la oferta de alternativas de recreación, las niñas optaban por mantenerse en la actividad, ya que, no sólo se contaba con la regulación de lo que las adolescentes debían hacer dentro de la sala de clase, sino, el que como monitora ofreciera en ocasiones una alternativa de esparcimiento, dentro del rol asignado, la historia y el contexto del centro, no daba opciones reales de ejercer este espacio de recreación, lo que estaba unido a las disposiciones arquitectónicas que visibilizaban a las alumnas fuera de sus aulas.

La regulación institucional hacia las adolescentes, en la búsqueda de un mejor desempeño educacional, no terminaba ahí, se busca desarrollar en estas adolescentes, valores morales y para esto la institución contaba con que las monitoras, no sólo ejercieran la labor educativa, sino que ellas mismas se constituyan como "modelo-referente"hacia las adolescentes. Cito una frase del diario de campo: "El refuerzo escolar es algo más que ayudar a los deberes, es también un referente, dando hábitos de estudio y hábitos de vida". Por lo que se plantean una orientación más allá de los objetivos educacionales, que por medio de las monitoras tengan un modelo de "dar en gratuidad y un referente para sus vidas".

Estas expectativas ejercían una regulación sutil acerca del comportamiento esperado de las monitoras, por ejemplo, el ocultamiento de ciertas acciones como fumar, frente al grupo de adolescentes. Permitían a su vez, una internalización de este dispositivo en las monitoras al definirse así mismas -una de ellas- como un "modelo normalizado cercano".Así a través de sus comportamientos, la monitora buscaba ser un modelo de conducta a seguir para las adolescentes, esto en el supuesto, de que los otros modelos presentes en la vida de las adolescentes no eran adecuados y que por su desviación y anormalidad se debía contrarrestar su influencia. En la efectividad de este dispositivo cobrara un importante papel la temporalidad y frecuencia de las actividades.>

El dispositivo "modelo-referente" se extendía a la relación padres-hijas de modo que la institución esperaba que los padres se volvieran modelo de conductas y valores esperados, frente a sus hijas. Algunas de las orientaciones en las charlas de padres recurrían frecuentemente al "modelo-referente", "que si los padres leen los niños también leerán", y así, este modelo se ofrecía como solución a la diversidad de problemáticas que presentaban los padres. 
La constitución del dispositivo "modelo-referente" se vuelve un efectivo sistema que regula tanto a las adolescentes, monitoras, padres e institución. Este modelo se inscribe en un uso particular de los espacios (arquitectura), y en el uso provechoso de los tiempos (rutinas y horarios) y actividades (que respondan a los valores de la institución).

\section{Prácticas Disciplinarias}

Las estrategias de gobierno, no sólo se ejercían dentro de los centros donde asistían las adolescentes, sino que se extendían a la familia y las redes institucionales. Por medio de una "des-gobermentalización del Estado" dado que el control era distribuido en diferentes actores sociales y voluntarios para su ejercicio, sin dejar de estar verificadas ni supervisados por éste. ${ }^{(8)}$.

La coordinación con servicios sociales y las escuelas en uno de los centros, permitió obtener información íntima desde "las expertas" (asistentes sociales, profesoras, etc.) acerca de las problemáticas psicosociales que vivían las adolescentes.

Dicha información, volvía a las adolescentes altamente vulnerables, ya que desde el lenguaje de los expertos se les catalogaba y subjetivaba en categorías de exclusión y marginalidad, lo que difícilmente permitiría obtener otras lecturas de la situación personal y familiar descrita. Por otra parte, esta coordinación con la red institucional, limitó "los usos" que hacían algunas familias, ya que al no estar coordinados los centros entre sí, algunas familias podían obtener beneficios de más de un centro. Esta coordinación comenzó por medio de reuniones y la entrega de un listado de las niñas y adolescentes del centro, a los servicios sociales. De este modo, las prácticas de confesión"(i) , no se realizaban desde una interrogación directa a las adolescentes, sino que se utilizaban mecanismos especializados de gobierno a distancia, siendo "los expertos" ${ }^{\text {"(8) }}$, los que describían y aportaban al centro "con todo detalle" la intimidad de las familias y de cada una de las integrantes, con esto me refiero a que se obtenía información de datos familiares, problemas, situación económica, dinámica familiar, etc.

La presencia de una micropenalidad en los centros de educación del tiempo libre, estaba dada en muchos detalles, y recorría la totalidad del dispositivo "modelo-referente". Involucraba las formas de comportarse tanto de las niñas y las monitoras- y la definición del rol de monitora como autoridad. Respecto de la conducta de las adolescentes se les demandaba regulaciones en sus comportamientos (por ejemplo, no decir malas palabras en la calle). A las monitoras en cambio, se les demandaba un desempeño adecuado del rol, expresado en aspectos como la puntualidad, la pertinencia de las actividades, rol de autoridad, etc.

\section{Categorías que guían la intervención}

La educación del tiempo libre se apoyaba en la categoría de adolescencia, al aportar el desarrollo normalizado por etapas o edades, como una guía para la intervención, separación de grupos y selección de actividades. Esto permitía la valoración de las adolescentes según su ajuste o no a ellas: "jugar a las compras y madres y padres a los 13 años es súper infantil "(ii). También se evaluaba la conducta de las adolescentes desde aspectos de género y culturales.

Paralelamente la marginalidad, o sea el entendimiento de las adolescentes como personas pobres y carentes, era una manera de entender a las adolescentes, que las posicionaba en falta. En general, la educación del tiempo libre esta traspasada por una lectura, de que las capacidades de las adolescentes están disminuidas y en ello colabora una psicologización de las capacidades y/o limitaciones que presentan las adolescentes. $\stackrel{(12)}{\text { en }}$

Esta valoración se vincula con la lógica de la caridad y el dar al que no tienen. Por lo tanto la lectura del otro como marginal, lo sitúa en posición de recepción de la ayuda ofertada, "tenemos lo que tenemos, trabajamos con gente pobre en muchos sentidos". (xiii)

Hacia que sujeto se perfila la educación del tiempo libre: 
Tanto las prácticas disciplinarias, como las categorías que guían el proceso educativo en el tiempo libre, buscan que emerja desde el niño la capacidad de auto dirigirse, en base a los valores propuestos. Como dice Vaughan ${ }^{(16)}$ se busca cultivar "una subjetividad más activa" que permita que la gente joven tome más responsabilidad por sus vidas.

Esto se relaciona con la concepción de la adolescencia como una realidad problemática y el tiempo libre como una amenaza, y que por tanto, es necesario su control e intervención. Así, el ideal de autogestión y participación de las adolescentes se ve atravesado por las prácticas disciplinarias y el deseo de formar individuos "útiles" a la sociedad y con valores morales determinados de acuerdo a un ideal preexistente de persona.

Así, el sujeto adolescente en la educación en el tiempo libre, está cruzado por diferentes discursos y prácticas que le constituyen. Es posible ver, como están presentes aspectos del desarrollo, culturales, socioeconómicos, etc. Entendemos estos como partes del contexto y la situación, tendrán mayor o menor densidad frente a otros aspectos e incidirán en la subjetivación de las adolescentes.

Sin embargo, aún cuando ciertas categorías marcaban los procesos de subjetivación de las adolescentes, siempre éste es un juego de agencia y resistencia. Por ejemplo, aún cuando la participación oficial de las adolescentes cubría escasos ámbitos, en muchas ocasiones las actividades debían negociarse con el grupo de adolescentes para llevarlas a cabo. A su vez, algunas adolescentes contaban con un mayor control de la situación, ya que muchas de ellas llevaban más años que las monitoras, participando en el "Casal", y conocían mejor el funcionamiento y reglas.

El hacer las cosas de maneras diferentes por parte de las adolescentes, era una práctica cotidiana y sutil de resistencia. Por ejemplo, hacer sus tareas de otra forma, el apoyo y complicidad que se daban entre sí para evitar responsabilidades, el vínculo estratégico entre ellas y la lealtad entre amigas, eran algunos de los

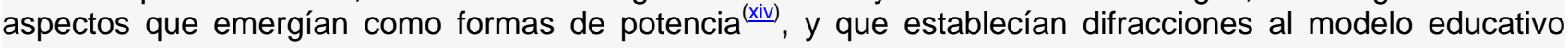
planteado.

Por ejemplo, en uno de los grupos, el funcionamiento dependía del liderazgo que ejercía una de las adolescentes del grupo. La dinámica de "complicidad con el liderazgo" que mantenía la monitora con la líder, regulaba muchas de las actividades, por lo que era necesaria para el desarrollo de las rutinas del "Casal".

La incorporación de esta relación "complicidad" por parte de la monitora encargada puede ser entendida desde dos puntos de vista, por un lado refleja como la monitora en su rol institucional reconoce e utiliza las estrategias del grupo para llevar a cabo sus objetivos educacionales; y por otro, como un posibilidad de resistencia por parte de las adolescentes, a las clásicas formas de enseñanza y su aceptación sólo desde la situación lúdica. En estas dinámicas podemos observar cómo la gobernabilidad se da en un espacio de libertad, entre unas y otras..

Cabe destacar, que estos aspectos eran más notorios en el centro que se encontraba reorganizándose y mucho menor en el centro que tenía más años de funcionamiento. Esto nos lleva a considerar las posibilidades de agencia/potencia en instituciones más o menos consolidadas, que aquí llamaremos más o menos densas.

(x) El texto original se apoya en las notas de campo. En este artículo y por las limitaciones de espacio no he vinculado estas notas, que aportan dinamismo y mayor comprensión al texto.

(xi) Cuando hablo de prácticas de confesión me refiero al uso que da Michel Foucault. Acerca de cómo se ha institucionalizado a lo largo de la historia, esta práctica religiosa, -de contar con "todo detalle" los actos personales a otro-, como forma de regulación. Y esto, ocurría en los centros de educación de tiempo libre, cuando no se consultaba de modo directo a la adolescente, sino se obtenía la información por medio de los expertos que han consultado previamente a la familia y redes. 
(xii) nota del diario de campo

(xiii) nota del diario de campo

(xiv) "La potencia desborda al poder que la habilita. Podría decirse que los propósitos del poder no siempre coinciden con los propósitos de la potencia. En la medida que estos últimos divergen de los primeros, la potencia supone la asunción de un propósito no pretendido por el poder." (17)

\section{" Conclusiones "}

Llegado a este punto y recordando las motivaciones iniciales del presente trabajo de investigación, iremos colocando atención a cada una de las principales inquietudes que guiaron este trabajo, con el deseo de generar "comprensiones, responsables, comprometidas y prometedoras" $\left.{ }^{13}\right)$ de cara al trabajo que hemos realizado y posiblemente seguiremos realizando con las adolescentes.

Las preguntas iniciales de cómo un ámbito definido por la autonomía y la autogestión, contiene formas de "subjetivación" y "gobernabilidad" hacia las adolescentes, las podemos dialogar con los siguientes aspectos:

¿Hay tiempo libre?

Al comienzo del trabajo de investigación, nos preguntamos acerca de cómo se gestiona el tiempo libre de las adolescentes, en este momento consideramos necesario distinguir claramente entre "tiempo libre" y la "educación en el tiempo libre". Así, un espacio definido por su libre albedrío, la posibilidad de ocio, de desarrollo de juegos y recreación, que iba desde nuestra salida de la educación formal, hasta la hora de dormir, se ha visto cada vez más colonizado por esta "educación en el tiempo libre", que se apropia del "tiempo libre" y lo gestiona.

La apropiación institucional que se hace del "tiempo libre", es importante dado su poder performativo ${ }^{(\underline{x v})}$, (que al ser utilizado y/o dicho, se actúa y produce efectos) ya que convierte un espacio de juego, no reglamentado, desorganizado y no tutelado, en un espacio para la formación de capacidades y destrezas, corrección de actitudes y educación en valores. Para luego, enmarcarlo nuevamente, dentro del concepto previo de libertad; gestionando los espacios de "libertad" de las adolescentes, para su uso provechoso.

De este modo, el tiempo libre ha sido reducido a su mínima expresión, extendiendo cada vez más un mayor control de la actividad -minuto a minuto. Es así como, progresivamente, este tiempo ha sido habitado por formas educativas, (juegos didácticos, programas televisivos educativos, aprendizaje musical, desarrollo de deportes, etc.). Hoy no es posible tener tiempo sin actividad y no hay actividad sin un fin, la experiencia temporal actual considera al tiempo como una mercadería ${ }^{(14)}$.

Cardús ${ }^{(14)}$ ya en 1985 , indicaba que la preocupación por la rentabilidad de ésta mercadería -el tiempo- ha generado acciones educativas que eviten su mal uso y su correcta inversión. Así, la principal problematización que se hace del "ocio" es su ocupación, lo que hace sentir como una "necesidad" el "educar el tiempo libre".

Por lo tanto, las generaciones juveniles se desarrollan en una optimización de su haceres en pro de su futuro -del cual sólo vislumbramos su precariedad- así, mientras más habilidades desarrolladas, más posibilidades. Entonces, la "educación en el tiempo libre" se expresa como la extensión máxima de los servicios educativos a la vida de las niñas y adolescentes.

Múltiples categorías

Múltiples categorías intervienen en la "educación en el tiempo libre", cuando es leída la normalidad y/o anormalidad de quienes en ella participan. En la presente investigación, ha sido posible vislumbrar la forma 
en que las lecturas de las adolescentes se dan desde aspectos del desarrollo (adolescencia), cultura y situación socioeconómica. Sin embargo, los entendimientos acerca de las adolescentes en situación de marginalidad y pobreza, cobran especial relevancia y calzan con la idea de adolescente "en falta".

La "educación en el tiempo libre" está traspasada por una lectura de que las capacidades de las adolescentes están disminuidas ${ }^{(12)}$ y en ello colabora una psicologización de las capacidades y/o limitaciones que presentan las adolescentes.

Así, la valoración "en falta", enmarcada en categorizaciones psicológicas, sociológicas y económicas, permite un entendimiento de las adolescentes que simplifica y unifica la complejidad de las situaciones en las que se desarrolla la vida de cada una de las adolescentes, y a su vez, calza con la lógica de la caridad, al dar al necesitado.

Este entendimiento, promueve que las dificultades del proceso educativo sean puestas en las adolescentes y no en quienes gestionan las actividades.

Qué tipo de sujeto (adolescente) desea configurar la educación en el tiempo libre.

La educación en el tiempo libre, se basa sobre el ideal del "niño libre", que explora sus capacidades y construye su futuro, con el objetivo de que, desde "él mismo" se desarrolle el ideal de persona esperado, promoviendo su agencia y autogestión, en la lógica que la institucionalidad espera.

Rose ${ }^{(15)}$ habla acerca de una nueva definición de los sujetos de gobierno, en cuanto sujetos activos que participan en su propio gobierno, que no necesitan ser gobernados, sino que se gobiernan y controlan por sí mismos, y se cuidan solos. Así, el buen sujeto de gobierno se fusionara con obligaciones asumidas voluntariamente por individuos "libres", con el objeto de sacar el mayor provecho de su propia existencia, mediante la gestión responsable de su vida.

Consideramos que en la "educación del tiempo libre", al igual como ocurrió con la implantación del desarrollo como pedagogía ${ }^{(20)}$, sucede un desplazamiento similar. En la búsqueda de un ideal de persona útil a la sociedad, se ha generado una educación continua, en que las actividades son predefinidas por la institución en base al modelo de persona que se quiere formar. Así, el discurso se mueve dentro de dos facetas, por un lado se promueve el libre albedrío de las adolescentes, y por otro se busca que hagan ocupaciones útiles a los ojos de la institución.

El modelo educativo da pocas opciones a la elección, a la toma de decisiones, al desarrollo de los intereses y actúa sobre un sujeto adolescente pasivo, desde su valoración "en falta", aún cuando, en las definiciones y orientaciones de la "educación del tiempo libre" se busca constantemente la participación y autogestión las iniciativas juveniles. Por lo tanto, se genera un estancamiento de la buscada "participación juvenil" debido a la excesiva burocratización de los procesos, escasa participación y realización de acciones "pensadas por otros expertos" alejadas de la situación concreta de las adolescentes.

Así, las adolescentes, dada su situación de "transición y crisis", son consideradas "cuidadanos condicionales" $\left.{ }^{\prime 16}\right)$ que tendrán que desarrollar, -por medio del "proceso de civilización "(vi), una subjetividad activa y responsable con el fin de lograr su "posible" inserción "responsable" a una sociedad precarizada. Entonces, lo que se busca es producir trabajadores adaptables que tengan la capacidad mental de ajustarse al no anunciado cambio causado por la tecnología ${ }^{(16)}$.

(xv) Performatividad es terminología que se deriva del inglés performance, que quiere decir actuar o interpretar. Su uso en el estudio se refiere a las diferentes categorías que son "actuadas" o "interpretadas" por las adolescentes, o cómo son consideradas por las otras. 
(xvi) Norbert Elias (1994 citado en Vaughan, 2000) desarrolló la idea de un 'proceso de civilización', entendiéndolo como el incremento de la regulación y sujeción de la conducta de todos, a través del crecimiento de redes complejas de interdependencias.

\section{" Referencias =}

(1) Walkerdine, V. (1992). Mujeres de clase obrera. Aspectos psicológicos y sociales de supervivencia. En F. Álvarez-Uría (Ed.), Marginación e Inserción. Madrid: Endymión.

(2) Trilla, J. (1993). La educación fuera de la escuela. Ámbitos no formales y educación social. Barcelona: Editorial Ariel S.A. (1998).

(3) Spink, P. (2004, Junio). "Más allá de la psicología organizacional". Seminario psicología social actual, Universidad Autónoma de Barcelona, Barcelona, España.

(4) FIC. (2004). Los sentidos de la crítica. Fractalidades en Investigación Crítica. En trámite de publicación, Athenea Digital, Revista de Pensamiento e Investigación Social.

(5) Pujol, J. (1999). Decontructing and reconstructing: producing a reading on "human reproductive technologies". En C. Willing (Ed.) Applied discourse analysis: social and psycological ilntervention. (pp. 87- 109). Buckinghan: Open University Press.

(6) Velasco, H. y Díaz de Rada, A. (1997). La Lógica de la investigación etnográfica. Madrid: Editorial Trotta, S.A.

(7) Guarderas, M. P. y Gutiérrez, P. (2004). Diálogos sobre identidad y sus implicancias metodológicas. Trabajo del curso Fractalitat en Investigació Critica II. Programa de doctorado en psicología social de la UAB. Barcelona.

(8) Rose, N. (1997). El gobierno en las democracias liberales: del liberalismo al neoliberalismo. Revista Archipiélago, cuadernos de crítica de la cultura, (29), verano, 2540.

(9) Rossiter, A. (2000). The postmodern feminist condition. En B. Fawcett, B. Featherstone, J. Fook y A. Rossitier, Practice and research in social work (pp. 24-38). London: Routledge.

(10) Rose, N. (1989). Chapter eleven: the young citizen. En N. Rose, Governing the soul. The shaping of private self. (pp. 121-131). London: Routledge.

(11) Montenegro, K. (2004). El capítulo de la mirada, epistemologías, teorías, reflexiones y prácticas. Trabajo de curso Fractalitat en Investigació Critica II. Programa de doctorado en psicología social de la UAB. Barcelona.

(12) Hendrick, 1990:210 citado en Vaughan, 2000. Vaughan, B. (2000). The Goverment of youth: disorder and dependence?. Social \& Legal Studies, 9(3), 347-366.

(13) Escuela de Otoño. (2004). Mesa de Experiencias: "Investigación para la acción". V Escuela de Otoño, Escuela Pública de Animación y Educación en el Tiempo Libre y Juvenil. Dirección General de la Juventud. Comunidad de Madrid. España.

(14) Cardús, S. (1985). El tiempo lliure. En Cardús, Miranda, Franch, Gassó, Verdú, Armengol y Navarro. Jornades catalanes d' educació en el temps lliure. Ponències. (pp. 5-15) 
Barcelona: Entitats d'educació en el temps lluire de Catalunya.

(15) Rose, N. (1996). Inventing ourselves. Psychology, power, and personhood. United Kingdom: Cambridge University Press. 1998.

(16) Vaughan, B. (2000). The Goverment of youth: disorder and dependence?. Social \& Legal Studies, 9(3), 347-366.

(17) Butler, J. (1997). Introducción. En J. Butler (1997), Mecanismo psíquicos del poder. Teorías de sujeción (pp. 11-41) Madrid: Ediciones Cátedra. (2001).

(18) Medrado, B. (2002). Tempo ao Tempo. A gestao da vida em idade. Tese doutorado. Sao Paulo: Pontificia Universidade Católica de Sao Paulo. (PUC).

(19) Foucault, M. (1991). Chapter Four: Govermentality. En G. Burchell, C. Gordon y P. Miller (Ed.), The Foucault effect. Chicago: The University of Chicago Press.

(20) Walkerdine, V. (1995). Psicología del desarrollo y pedagogía centrada en el niño. La inserción de Piaget en la educación temprana. En J. Larrosa (Ed.), Escuela, Poder y Subjetivación (pp. 79-152). Madrid: Ediciones de La Piqueta. 